\title{
Resistencia natural de trece maderas mexicanas al ataque de termitas subterráneas
}

\author{
Ricardo Reyes Chilpa ${ }^{1}$ \\ Nohemí Viveros Rodríguez ${ }^{1}$ \\ Víctor Pérez Morales ${ }^{2}$
}

\section{RESUMEN}

Se evaluó la resistencia natural del duramen de trece especies forestales (seis de selva alta perennifolia y siete de bosque de pino-encino) al ataque de termitas subterráneas Heterotermes sp. mediante micropruebas de campo. Bloques pequeños $(2.5 \times 2.5 \times 5 \mathrm{~cm})$ de cada especie se colocaron en cuatro rejillas de madera susceptible siguiendo un diseño de cuadrado latino. Las rejillas se colocaron en el suelo de una selva tropical caducifolia, en el Centro de Investigaciones Costeras, La Mancha, Veracruz, México. La duración total de la prueba fue de 15 meses; los resultados se estimaron visualmente de acuerdo con la magnitud del daño sufrido, estableciéndose cinco categorías. En general las maderas de la selva alta perennifolia fueron mas resistentes que las del bosque de pinoencino. Para algunas especies se observaron diferencias en el grado de ataque que sufrieron entre las cuatro rejillas; se discuten las posibles causas de esta variabilidad. Las clasificación de las maderas fue la siguiente: Muy Resistentes: Guarea chichon, Swietenia macrophylla (Meliaceae); Quararibea funebris (Bombacaceae). Resistentes: Pinus patula var. longepedunculata (Pinaceae); Lysiloma acapulcensis (Leguminosae). Poco Resistentes: Pinus patula, Pinus aff. pseudostrobus (Pinaceae); Quercus acatenangensis (Fagaceae). Susceptibles:

\begin{abstract}
Ninguna. Muy Susceptible: Quercus crassifolia (Fagaceae); Alnus jorullensis (Betulaceae). Para Pinus teocote (Pinaceae); Schizolobium parahibum (Leguminosae) y Licania platypus (Rosaceae) no se obtuvieron resultados concluyentes.
\end{abstract}

\section{PALABRAS CLAVE}

Duramen, resistencia natural, termitas, micropruebas de campo, cuadrado latino, Heterotermes sp.

\section{ABSTRACT}

The natural resistance of heartwood from thirteen forest species (six of Tropical Rain Forest and seven of Pine-Oak Forest) to subterranean termites Heterotermes sp. attack, was evaluated by field microtests. Small blocks $(2.5 \times 2.5 \times 5 \mathrm{~cm})$ of each species were settled in 4 racks made of a highly susceptible wood, following a latin square arrangement. The racks were placed on the soil of the test area, in a tropical deciduous forest of the Biological Station "La Mancha", Veracruz, México. The total exposure period was 15 months. Natural resistance was evaluated by means of termite damage, according to a visual-rating system with five categories. Tropical rain forest woods were more resistant that pine-oak forest woods. For certain species, differences in the rank of attack were found between raks, possible causes of this variability are discussed. The classification of woods was:

1 Investigador y asistente de investigador, respectivamente. Departamento de Productos Forestales y Conservación de Bosques. Instituto de Ecología A.C. Apdo. Postal 63. Xalapa, Ver., 91000 México. $\quad{ }^{2}$ Chapas y Maderas Importadas, S.A. México, D.F. Manuscrito recibido para su publicación el 30 de octubre de 1993. 
Highly Resistant: Guarea chichon, Swietenia macrophylla (Meliaceae); Quararibea funebris (Bombacaceae).

Resistant: Pinus patula var. longepedunculata (Pinaceae); Lysiloma acapulcensis (Leguminosae).Moderately Resistant: Pinus patula, Pinus aff. pseudostrobus (Pinaceae); Quercus acatenangensis (Fagaceae). Susceptible: None. Highly Susceptible: Quercus crassifolia (Fagaceae); Alnus jorullensis (Betulaceae). In the case of Pinus teocote (Pinaceae); Schizolobium parahibum (Leguminosae) and Licania platypus (Rosaceae) no conclusive results were obtained.

\section{KEY WORDS}

Heartwood,natural resistance, termites, field microtests, latin square, Heterotermes sp.

\section{INTRODUCCION}

La madera es un material orgánico atacado por agentes biodegradadores, entre los que se encuentran las termitas. Estos insectos son capaces de causar grandes pérdidas económicas, las cuales han sido calculadas en 1500 millones de dólares para Estados Unidos de América (Ebeling, 1968). En las zonas tropicales, dichas pérdidas son aún más severas, pero no existen cifras confiables al respecto. Los daños por termitas pueden, sin embargo, prevenirse mediante mediadas adecuadas de diseño, tratamientos químicos y selección de los materiales (Pérez, et al, 1981). En relación a este último aspecto, se saben que existen maderas que se caracterizan por su resistencia natural al ataque de estos organismos. Esta propiedad se debe principalmente a la presencia de metabolitos secundarios tóxicos y/o repelentes a las termitas, llamados comúnmente extractivos, en las paredes y lúmenes celulares (Carter et al, 1982; Carter et al, 1975; Carter et al, 1983; Scheffrahn, 1991). Para evaluar la resistencia natural de la madera a las termitas se han diseñado diferentes métodos de prueba, tanto de campo (Howick et al, 1983) como de laboratorio
(Beal, 1976; Pérez et al, 1984). En este estudio se empleó el método propuesto por Williams (1973), con algunas modificaciones, debido a que ofrece las siguientes ventajas:

a) El desarrollo de la prueba se realiza en el campo en condiciones naturales, por lo que no se altera el comportamiento de las termitas.

b) Se pueden utilizar especies de termitas que son difíciles de mantener en el laboratorio por mucho tiempo.

c) Se pueden seleccionar las especies de termitas que se deseen estudiar de acuerdo a su distribución en el área.

d) Se obtienen resultados en un lapso relativamente corto.

Dicho método requiere, sin embargo, el reconocimiento previo del área de pruebas, primordialmente de las termitas presentes. Los objetivos de este estudio fueron: a) evaluar la resistencia natural de 6 maderas tropicales y 7 de bosque de pino-encino al ataque de termitas subterráneas, b) examinar el desempeño del método de Williams (1973), así como de las modificaciones aquí propuestas. Los resultados obtenidos ofrecen un índice de las resistencia natural de las maderas estudiadas. Estos índices, así como otros obtenidos para termitas de madera seca, pueden orientar a los usuarios en la selección de maderas dependiendo de las zonas de riesgo al ataque de estos organismos que se han descrito para México (Pérez et al, 1984).

\section{METODOLOGIA}

Las maderas estudiadas se obtuvieron de la colección de LACITEMA. El material de ensayo consistió en bloques de duramen de $2.5 \times 2.5 \times 5 \mathrm{~cm}$ (la mayor dimensión paralela a la dirección de la fibra). Seis de las especies fueron de selva alta perennifolia (Selva Lacandona, Chiapas, México) y siete de bosque de pino-encino (Sierra Juárez, Oaxaca, México) (Tabla 1). 
Tabla 1. Especies estudiadas

\begin{tabular}{|c|c|c|c|}
\hline ESPECIES & $\begin{array}{l}\text { NOMBRE } \\
\text { COMUN }\end{array}$ & REGISTRO ${ }^{\mathrm{a}}$ & $\underset{b}{C}$ CLAVE \\
\hline \multicolumn{4}{|l|}{ SELVA ALTA PERENNIFOLIA } \\
\hline Guarea chichon C.D.C. (MELIACEAE) & Cedrillo & 56 & $\mathrm{G}$ \\
\hline Licania platypus (Hemsl.) Fritsch (ROSACEAE) & $\begin{array}{l}\text { Cabeza de } \\
\text { Mico }\end{array}$ & 57 & Lp \\
\hline Lysiloma acapulcensis (Kunth) Benth (LEGUMINOSAE) & Tripal & 44 & La \\
\hline Quararibea funebris (Llave) Vischer (BOMBACACEAE) & Molinillo & 54 & $\mathrm{~F}$ \\
\hline Schizolobium parahibum (Vell) Blake (LEGUMINOSAE) & Guanacastle & 35 & $S$ \\
\hline Swietenia macrophylla (King) (MELIACEAE) & Caoba & 37 & Sw \\
\hline \multicolumn{4}{|l|}{ BOSQUE DE PINO-ENCINO } \\
\hline Alnus jorullensis H. B. K. (BETULACEAE) & llite & 104 & A \\
\hline Quercus acatenanguesis (trel) (FAGACEAE) & Encino & 95 & Qa \\
\hline Quercus crassifolia H.B. (FAGACEAE) & Encino & 90 & Qc \\
\hline Pinus patula Schl. Et Chan (PINACEAE) & Pino & 102 & $P$ \\
\hline $\begin{array}{l}\text { Pinus patula var. longepedunculata } \\
\text { Schl. Et Chan (PINACEAE) }\end{array}$ & Pino & 105 & $\mathrm{PI}$ \\
\hline Pinus aff pseudostrobus Lind (PINACEAE) & Pino & 99 & $\mathrm{Pp}$ \\
\hline Pinus teocote (Schl. Et Chan) (PINACEAE) & Pino & 101 & $\mathrm{Pt}$ \\
\hline
\end{tabular}

Xiloteca "Dr. Faustino Miranda" Instituto de Ecología, A.C.

Claves de las especies empleadas en las figs. 2, 3, 4 y 5

El estudio se realizó en el Centro de Investigaciones Costeras, La Mancha, localizada en el Estado de Veracruz, México. Presenta un clima sub-húmedo con lluvias en verano (AW ), con una precipitación entre 1200 y $1500 \mathrm{~mm}$ anuales; las temperaturas máxima y mínima extremas son $34^{\circ} \mathrm{C}$ y $16^{\circ} \mathrm{C}$ respectivamente, y la temperatura media anual es de $24^{\circ} \mathrm{C}$. La vegetación en el área de prueba consiste en Selva Baja Caducifolia (Novelo, 1978).

La resistencia de las maderas se evaluó mediante las micropruebas de campo (Williams, 1973) con algunas modificaciones. La innovación más importante fue el empleo de una rejilla de madera como soporte de los bloques de prueba (fig. 1) y para asegurar el acceso de las termitas desde cualquier dirección. Las rejillas se fabricaron con tiras de madera de Alnus jorullensis, ya que por observaciones previas de detectó que esta madera es muy susceptible al ataque de termitas, tanto de madera seca como subterráneas.

Se prepararon tres rejillas con capacidad para contener 25 bloques de prueba. Adicionalmente se preparó otra rejilla con capacidad para 49 bloques. Los bloques se colocaron en las rejillas siguiendo un diseño de cuadrado latino. En el caso de las rejillas pequeñas éste fue de $5 \times 5$ (cinco réplicas 


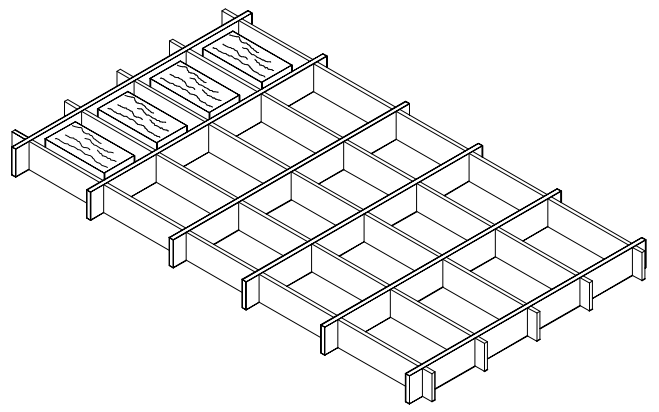

\begin{tabular}{|c||c||c||c||c|}
\hline $\mathrm{E}$ & $\mathrm{D}$ & $\mathrm{C}$ & $\mathrm{B}$ & $\mathrm{A}$ \\
\hline \hline$\Delta$ & $\Delta$ & $\Delta$ & $\Delta$ & $\mathrm{B}$ \\
\hline \hline & & & $\Delta$ & $\mathrm{C}$ \\
\hline & & & $\Delta$ & $\mathrm{D}$ \\
\hline \hline & & & $\Delta$ & $\mathrm{E}$ \\
\hline \hline
\end{tabular}

Figura 1. Rejilla y distribución de los bloques

de cinco especies diferentes) y para la rejilla grande fue de $7 \times 7$. En ningún caso se repiten bloques de la misma especie en cada columna y fila (Fig. 1) . Cada uno de los cuadros latinos de $5 \times 5$ incluyó bloques de especies diferentes, exceptuando $A$. jorullensis que fue la de referencia (Williams, 1973). En el cuadrado latino de $7 \times 7$ se incluyeron bloques de seis de las especies presentes en los cuadrados anteriores, además a Alnus jorullensis. Las micro- pruebas se distribuyeron dentro del área de prueba (superficie aproximada $500 \mathrm{~m}^{2}$ ), en sitios donde de acuerdo con muestreos previos existía una mayor abundancia de termias subterráneas Heterotermes sp. (Williams, 1980).

La instalación de las micropruebas se realizó de la siguiente manera: se depositaron dos tabiques en el suelo cubiertos por dos placas de vidrio, a fin de aislar la prueba de la humedad y evitar el ataque por hongos. Luego se colocó una de las rejillas con los bloques y se cubrió con dos placas de vidrio. En las esquinas de los tabiques se instalaron varillas de $A$. jorullensis de $1.5 \times 0.5 \mathrm{~cm}$ para atraer a las termitas. Las micropruebas fueron protegidas de la lluvia con una cubierta de tela plástica impermeable sostenida por un marco de fierro. Para aislarla de otros animales se rodeó con tela de alambre (malla de $0.5 \mathrm{~cm}$ ).

La duración total de la prueba fue de 15 meses, realizándose revisiones periódicas cada cuatro meses. Al final de este período se evaluó la resistencia natural de los bloques por medio de rangos visuales de daño (RVD) (Tabla 2). De acuerdo con los criterios establecidos por Williams (1973, 1980), se consideró que una microprueba había concluido y ofrecía resultados confiables, cuando un bloque de la madera de referencia

Tabla 2. Rangos visuales de daño (RVD)

\begin{tabular}{|c|l|c||}
\hline Calificación & \multicolumn{1}{|c|}{ Características } & Resistencia \\
\hline 1 & superficie roída o mordisqueada & muy resistente \\
\hline 2 & perforaciones poco profundas sobre una cara de los bloques & resistente \\
\hline 3 & $\begin{array}{l}\text { perforaciones poco profundas sobre la mayor parte de las } \\
\text { caras }\end{array}$ & poco resistente \\
\hline 4 & perforaciones profundas y galerías & susceptible \\
\hline 5 & gran porción de la madera destruída totalmente & muy susceptible \\
\hline
\end{tabular}


(A. jorullensis) había sido totalmente destruido (RVD $=5)$ y además el daño acumulado por todos los de referencia era mayor al $50 \%$ (RDV > 2.5). Los resultados de los cuadrados latinos (Tabla 3) se sometieron a un análisis de varianza (Mendenhall, 1982). Las termitas presentes en las rejillas y bloques de prueba, fueron identificadas por comparación con especímenes de la colección de termitas de LACITEMA. Por último se calcularon índices de resistencia natural para cada madera (Tabla 4). Para ello se empleó la suma de la media de los RVD y su correspondiente desviación estándar. Este procedimiento toma en consideración el máximo ataque registrado.

\section{RESULTADOS}

Después de 15 meses de exposición en el campo, el desarrollo de las micropruebas puede considerarse satisfactorio. Las termitas presentes fueron identificadas como Heterotermes sp. Las rejillas cumplieron el objetivo para el cual fueron diseñadas, pues constituyeron una excelente vía de acceso para las termitas. Numerosos tramos presentaron galerías y un buen número de éstos quedaron reducidos a las capas de madera mas externas. Otros fueron totalmente destruídos.

Tabla 3. Media y desviación estándar de los rangos visuales de daño de trece maderas expuestas a Heterotermes sp. en micropruebas de campo

\begin{tabular}{|l|c|c|c|c|c||}
\hline \multicolumn{1}{|c|}{ ESPECIES } & IV & II & III & I $^{*}$ & TOTAL \\
\hline Alnus jorullensis & $5.0 \pm 0.0$ & $4.0 \pm 1.5$ & $3.6 \pm 1.0$ & $1.8 \pm 0.9$ & $4.2 \pm 1.1$ \\
\hline Quercus crassifolia & $5.0 \pm 0.0$ & $3.6 \pm 1.0$ & - & - & $4.5 \pm 0.7$ \\
\hline Q. acatenanguensis & $2.8 \pm 0.9$ & - & - & $1.0 \pm 0.0$ & $2.8 \pm 0.9$ \\
\hline P. aff. pseudostrobus & $2.8 \pm 0.6$ & $1.4 \pm 0.4$ & - & - & $2.2 \pm 1.0$ \\
\hline Pinus patula & $1.7 \pm 1.0$ & - & $2.4 \pm 0.8$ & - & $2.0 \pm 1.0$ \\
\hline Lysiloma acapulcensis & $1.2 \pm 0.4$ & $2.0 \pm 1.5$ & & - & $1.5 \pm 1.1$ \\
\hline $\begin{array}{l}\text { Pinus patula variedad } \\
\text { longepedunculata }\end{array}$ & $1.2 \pm 0.4$ & - & $1.8 \pm 0.7$ & - & $1.5 \pm 0.9$ \\
\hline Quararibea funebris & - & - & $1.4 \pm 0.4$ & - & $1.4 \pm 0.4$ \\
\hline Guarea chichon & - & $1.0 \pm 0.0$ & - & - & $1.0 \pm 0.0$ \\
\hline Swietenia macrophylla & - & - & $1.0 \pm 0.0$ & - & $1.0 \pm 0.0$ \\
\hline Pinus teocote & - & - & - & $1.8 \pm 0.7$ & - \\
\hline S. parahibum & - & - & - & $1.6 \pm 0.4$ & - \\
\hline Licania platypus & $35 \mathrm{~s}$. & $11.7 \mathrm{~s}$. & $9.8 \mathrm{~s}$. & $1.2 \mathrm{n.s}$. & - \\
\hline Anava F( $\alpha=0.01)$ & & & - & $1.2 \pm 0.4$ & - \\
\hline \hline
\end{tabular}

* Datos excluídos del análisis

s. = diferencias significativas 
n.s. $=$ diferencias no significativas

Las micropruebas II, III y IV cumplieron con los criterios de conclusión y confiabilidad establecidos (cf. metodología). Para estas micropruebas el análisis de varianza confirmó que existen diferencias significativas en la resistencia de las maderas a las termitas (Tabla 3). La microprueba 1 (Fig. 2) no cumplió con dichos requisitos y sus resultados fueron excluídos del análisis; por tal motivo a tres de las maderas no se les asignó ninguna categoría de resistencia.

Microprueba I

Poco resistente

Figura 2. Microprueba 1 (claves de las especies en la tabla 1)

En las micropruebas II, III y IV la madera de referencia Alnus jorullensis fue la que experimentó el mayor ataque (Tabla 3).

En la microprueba IV todos los bloques de esta madera fueron totalmente destruídos (RVD = 5), pero en las micropruebas II y III sufrió un daño menor $(\mathrm{RVD}=4$ y 3.6, respectivamente) (Tabla 3). En promedio dicha madera fue clasificada como muy susceptible (Tabla 4), lo cual concuerda con nuestras observaciones previas.

Cinco de las maderas se evaluaron en dos micropruebas, para determinar si los resultados eran reproducibles. Pinus patula, Lysiloma acapulcensis y Pinus patula var. longepedunculata presentaron valores similares en ambas pruebas (Tabla 3). La primera especie fue clasificada como poco resistente, mientras que las últimas alcanzaron la categoría de resistente (Tabla 4). Pinus aff. pseudostrobus y Quercus crassifolia presentaron una mayor variabilidad en su comportamiento en las dos diferentes micropruebas (tabla 3 ).

\section{Microprueba II}

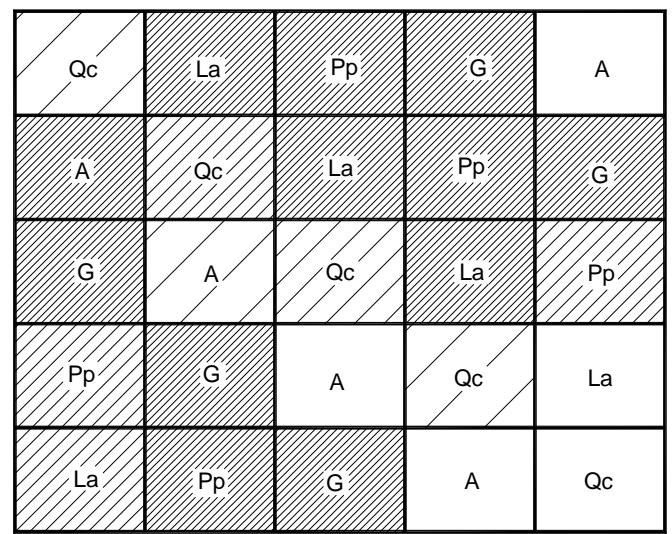

Figura 3. Microprueba II (claves en la tabla 1 y figura 2)

Microprueba III

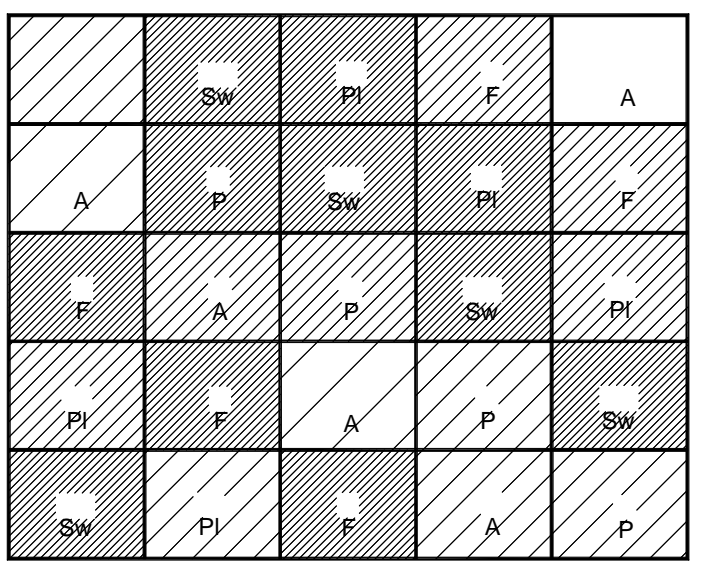

Figura 4. Microprueba III (claves en la tabla 1 y figura 2 ) 


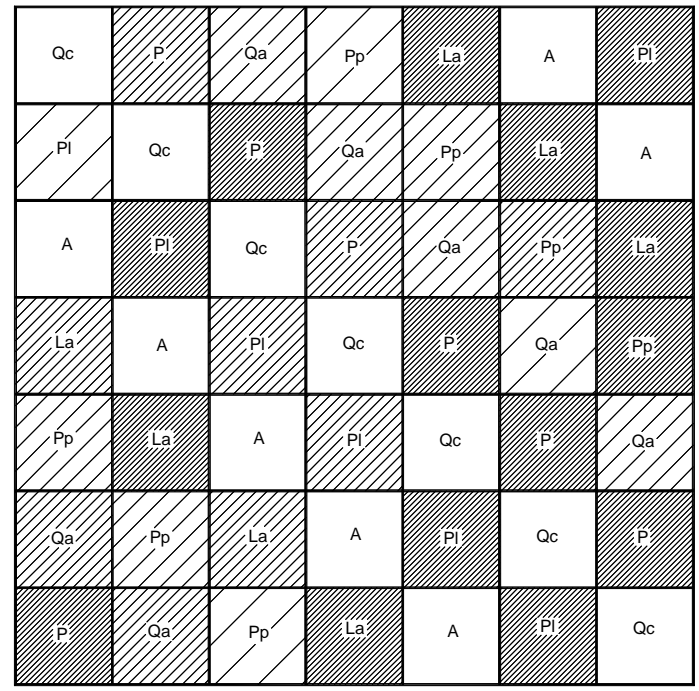

Figura 5. Microprueba IV (claves en la tabla 1 y fig. 2 )

Su clasificación final fue: poco resistente y muy susceptible, respectivamente (Tabla 4). Cabe señalar que Quercus crassifolia es comparable en susceptibilidad a Alnus jorullensis (Tabla 4 ).

Cuatro maderas solo fueron evaluadas en una sola microprueba. Quercus acatenanguensis resultó ser poco resistente, mientras que Guarea chichon, Swietenia macrophylla y Quararibea funebris fueron clasificadas como muy resistentes (Tabla 4).

\section{DISCUSION}

La existencia de preferencias alimenticias en pruebas de selección con diferentes maderas, ha sido demostrada en condiciones de campo y laboratorio, tanto con termitas subterráneas (Carter et al, 1982 y 1974; Williams, 1973), como con termitas de madera seca (Rust et al, 1977); y se atribuye principalmente a la presencia de extractivos tóxicos 0 repelentes a estos organismos (Scheffrahn, 1991).

Para que dichas preferencias se manifiesten, las condiciones de sobrevivencia de las termitas, y la duración de la prueba deben ser adecuadas. La naturaleza de las pruebas aquí empleadas, así como la infestación de las rejillas, sugieren que el primer factor fue satisfactorio. Considerando el ataque sobre la madera de referencia, el segundo factor sólo se logró plenamente en la microprueba IV (Fig. 5). Las otras micropruebas fueron atacadas, lo cual podría deberse a diferencias en la distribución de termitas en el área.

Puesto que en condiciones de campo no es posible controlar el número de organismos en cada prueba, sería necesario manipular su duración. Para lo cual es muy importante contar con una madera susceptible de referencia tal como lo hace

Tabla 4. Indices de resistencia de maderas mexicanas al ataque de termitas subterráneas Heterotermes sp.

Muy susceptibles : Alnus jorullensis (Betulaceae) y Quercus crassifolia (Fagaceae)

Susceptible: ninguna

Poco resistentes: Quercus acatenanguesis (Fagaceae), Pinus aff. pseudostrobus (Pinaceae) y Pinus patula (Pinaceae)

Resistentes: Lysiloma acapulcensis (Leguminosae), Pinus patula var. Longepedunculata (Pinaceae)

Muy resistentes: Quararibea funebris (Bombacaceae), Guarea chichon (Meliaceae) y Swietenia macrophylla (Meliaceae) 
notar Williams (1973). Nuestros resultados sugieren, que la conclusión de una microprueba de campo debe ser cuando todos los bloques de la madera susceptible de referencia alcance el máximo daño, ya sea que se estime visualmente, 0 por pérdida de peso de los bloques atacados.

No obstante que existe variabilidad en el grado de ataque para algunas especies por las causas antes mencionadas, es posible ofrecer ordenes de resistencia para 10 de las maderas estudiadas. Nuestros resultados no permiten señalar de manera preliminar que de las maderas estudiadas, el duramen de las especies de la selva alta perennifolia en mas resistente al ataque de Heterotermes sp. que el de las especies del bosque de pino-encino.

\section{CONCLUSIONES}

La microprueba de campo modificada mediante el empleo de una rejilla de madera susceptible al ataque por termitas es un método eficiente, rápido y económico para evaluar la resistencia natural de las maderas, al ataque de termitas subterráneas en condiciones de campo. A fín de asegurar que la infestación sea adecuada se recomienda retirar la microprueba cuando todas las muestras de la madera susceptible de referencia experimenten el máximo ataque. Mediante este método se determinó la resistencia de 10 maderas mexicanas al ataque de termitas subterráneas Heterotermes sp. En general las maderas de la alta selva perennifolia fueron mas resistentes que las de bosque de pinoencino.

\section{RECONOCIMIENTOS}

Agradecemos la colaboración del Biól. René Ramírez por su apoyo técnico, a los Ings. V. Rubén Ordóñez Candelaria, Mario Ricalde Camacho, Ignacio Salomón Quintana y Reyna Paula Zárate Morales por su ayuda con las ilustraciones.

\section{REFERENCIAS}

Beal, R.H. 1976. Resistance of tropical woods to subterranean termites. pp. 67-74. In: J.D. Bultman, ed. Proceedings of a Workshop on the Biodeterioration of Tropical Woods: Chemical Basis for Natural resistance. Naval Res. Lab. Dep. of The Navy. 2 Washington, D.C.

Carter, F. L. y R.H. Beal. 1982. Termite responses to susceptible pine wood treated with antitermitic wood extracts. The International Journal of Wood Preservation 2:185-191

Carter, F. L., R.H. Beal y J.D. Bultman. 1975. Extraction of antitermitic substances from 23 tropical hardwoods. Wood Science 8: 406410.

Carter, F.L. y C.R.R. Camargo. 1983. Testing antitermitic properties of Brazilian Woods and extracts. Wood and Fiber Science 15:350-357.

Carter, F.L. y R.V. Smythe. 1974. Feeding and survival responses of Reticulitermes flavipes (Kollar) to extractives of wood from 11 coniferous genera. Holzforschung 28: 41-45

Ebeling, W. 1968. Termites. Identification, biology and control of termites attacking buildings. University of California, Division of Agriclutural Sciences. Manual 38. EUA.

Howick, C.D. y J.W. Creffield. 1983. A rapid field bioassay group on wood preservation. IRG/WP Document 1188.

Mendenhall, W. 1982. Introducción a la probabilidad y estadística. Wadsworth International//beroamérica. Belmont, CA. EUA.

Novelo, R. A. 1978. La vegetación de la Estación Biológica El Morro de la Mancha, Veracruz. Biótica 3:9-23.

Pérez M., V., G. Bárcenas P. y R. Echenique M. 1981. Prevención y control de daño por termitas en estructuras con madera. La Madera y su Uso en la Construcción No. 7, INIREBLACITEMA, Xalapa, Ver. 
Pérez M., V. y R. Echenique M. 1983. Zonas en México donde se presentan termitas que dañan estructuras. Reporte final de actividades presentado al Fondo de Operaciones de Descuento Bancario a la Vivienda (FOVI), Banco de México.

Pérez M., V., G. Segura W. y J.L. Brunhuber M. 1984. Resistencia natural de 18 especies de maderas mexicanas contra el ataque de termitas de madera seca, Cryptotermes bervis, Walker (Kalotermitidae, Isóptera). I Congreso Mexicano de Patología Forestal, 26-29 Septiembre de 1984, Durango, Dgo. México.

Rust, M.K. y D.A. Reierson. 1977. Using wood extracts to determine the feeding preferences of the western drywood termite Incisitermes minor (agen). Journal of Chemical Ecology 3:391-399.

Scheffrahn, R.H. 1991. Allelochemical resistance of wood to termites. Sociobiology 19(1): 257-281.

Williams, R.M.C. 1973 Evaluation of field and laboratory methods for testing termite resistance of timber and building material in Ghana, with relevant biological studies. Centre for Overseas Pest Research, Tropical Pest Bulletin 3. Londres.

Williams, R.M.C. 1980. A visit to Mexico to advise on the termite research program at INIREB (LACITEMA) Xalapa, Veracruz, 7-29 November 1980 Report CVR/81/5. Manuscript of limited distribution. Centre for Overseas Pest Research. Londres. 